\section{( OPEN ACCESS}

\title{
The cellular composition of lymph nodes in the earliest phase of inflammatory arthritis
}

\author{
L G M van Baarsen, ${ }^{1,2}$ M J H de Hair, ${ }^{1}$ T H Ramwadhdoebe, ${ }^{1,2}$ IJ A J Zijlstra, ${ }^{3}$ \\ M Maas, ${ }^{3}$ D M Gerlag, ${ }^{1}$ P P Tak ${ }^{1,4,5}$
}

\begin{abstract}
Handling editor Tore K Kvien
- Additional material is published online only. To view please visit the journal online (http://dx.doi.org/10.1136/ annrheumdis-2012-202990).

${ }^{1}$ Division of Clinical Immunology and Rheumatology, Academic Medical Center, University of Amsterdam, Amsterdam, The Netherlands

2Department of Experimental Immunology, Academic

Medical Center, University of Amsterdam, Amsterdam, The Netherlands

${ }^{3}$ Department of Radiology, Academic Medical Center, University of Amsterdam, Amsterdam, The Netherlands ${ }^{4}$ University of Cambridge, Cambridge, UK

${ }^{5}$ GlaxoSmithKline,

Stevenage, UK
\end{abstract}

\section{Correspondence to} Dr L G M van Baarsen, Division of Clinical Immunology and Rheumatology,

Department of Experimental Immunology, Academic

Medical Center/University of Amsterdam, Meibergdreef 9 , Amsterdam 1105 AZ, The Netherlands; e.g.vanbaarsen@amc.uva.nl

LGMvB and MJHdH contributed equally.

Accepted 14 April 2013 Published Online First 9 May 2013

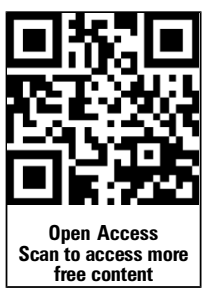

\section{ABSTRACT}

Objectives Rheumatoid arthritis (RA) is an immunemediated inflammatory disease of unknown aetiology. Recent work has shown that systemic autoimmunity precedes synovial inflammation, and animal models have suggested that changes in the lymph nodes may precede those in the synovial tissue. Therefore, we investigated the cellular composition of the lymph node in the earliest phases of inflammatory arthritis.

Methods Thirteen individuals positive for immunoglobulin M (lgM) rheumatoid factor and/or anticitrullinated protein antibodies without arthritis were included. Additionally, we studied 14 early arthritis patients (arthritis duration $\leq 6$ months, naive for diseasemodifying antirheumatic drugs), and eight healthy controls. All subjects underwent ultrasound-guided inguinal lymph node biopsy. Different T- and B-lymphocyte subsets were analysed by multicolour flow cytometry.

Results There was an increase in activated CD69 CD8 T cells and CD19 B cells in early arthritis patients compared with healthy controls. We also observed a trend towards increased CD19 B cells in autoantibodypositive individuals without arthritis compared with healthy controls.

Conclusions This exploratory study suggests that there is increased immune cell activation within lymph nodes of early arthritis patients as well as in autoantibodypositive individuals at risk of developing RA. This method provides a unique tool to investigate immunological changes in the lymph node compartment in the earliest phases of inflammatory arthritis.

\section{INTRODUCTION}

Rheumatoid arthritis (RA) is a prototypic inflammatory autoimmune disease with a poorly understood etiopathogenesis. Given the destructive nature of the disease, early diagnosis and start of treatment is highly important. ${ }^{1-3}$ Several studies have shown that elevated acute-phase proteins, chemokines, cytokines and RA-specific autoantibodies (rheumatoid factor (RF) and anticitrullinated protein antibodies (ACPA)) can be detected in peripheral blood years before the onset of arthritis. $^{4-9}$ In prospective cohort studies, these autoantibody-positive individuals can be defined as having systemic autoimmunity associated with RA and being at risk of developing $\mathrm{RA}^{10} \mathrm{~A}$ recent study showed that the cellular composition of the primary target of RA, the synovium, is comparable with that of healthy controls during this phase. ${ }^{11}$ Thus, systemic autoimmunity appears to precede the development of synovial inflammation. Since the RA-specific autoantibodies can be present for years without disease symptoms and without increased synovial cellularity, factors outside the synovial compartment should be responsible for the initial changes leading to RA.

As a general principle, the recruitment of activated immune cells to the site of inflammation is initiated after informing a nearby lymph node of a danger signal. Thus, the immune reaction in lymph nodes generally precedes the influx of effector cells into the target tissue. Indeed, animal models have shown that the onset of arthritis is preceded by phenotypic changes in the cellular compartment of draining lymph nodes, indicating a primary role for lymph nodes in the initiation of arthritis. ${ }^{12-14}$ However, very little is known about the initial events that occur in lymph nodes before disease onset in patients with arthritis. Recently, we developed core-needle biopsy sampling of inguinal lymph nodes for research in RA, and we have shown that the procedure is generally well tolerated. ${ }^{15}$ In the current study, we investigated the cellular composition of lymph node biopsies obtained from autoantibody-positive individuals at risk of developing RA, and compared the results with those observed in early arthritis patients and healthy controls.

\section{METHODS}

\section{Study subjects and lymph node biopsy sampling}

Individuals with elevated IgM-RF and/or ACPA levels without arthritis were included in the study. These individuals were otherwise healthy and have systemic autoimmunity associated with RA, and are therefore at risk of developing RA (phase c, ref. 10) (further referred to as 'at risk' individuals). Additionally, early arthritis patients (arthritis duration $\leq 6$ months, determined from the first clinical signs and symptoms of arthritis as assessed by the rheumatologist; disease-modifying antirheumatic drug naïve) and healthy controls without any joint complaints and without RA-specific antibodies were included. Ultrasound-guided inguinal lymph node biopsies were obtained by a radiologist using a 16G core needle as previously described, ${ }^{15}$ and immediately processed for flow cytometry analysis. The study was approved by the local ethical committee, and all study subjects gave written informed consent.

\section{Flow cytometry analysis}

Lymph node biopsy samples were put through a $70 \mu \mathrm{m}$ cell strainer (BD Falcon) to obtain a single 
Table 1 Demographic data of the study participants

\begin{tabular}{lccc}
\hline & $\begin{array}{l}\text { Healthy controls } \\
\mathbf{n = 8}\end{array}$ & $\begin{array}{c}\text { At risk individuals } \\
\mathbf{n = 1 3}\end{array}$ & $\begin{array}{c}\text { Early arthritis patients } \\
\mathbf{n = 1 4}\end{array}$ \\
\hline Sex, female (\%) & $5(63)$ & $10(77)$ & $9(64)$ \\
Age (years) (median (IQR)) & $32(28-44)$ & $48(34-54)$ & $54(34-60)$ \\
IgM-RF positive (n (\%)) & $0(0)$ & $5(39)$ & $7(50)$ \\
IgM-RF level* (kU/l) (median (IQR) & - & $265(79-1049)$ & $120(69-563)$ \\
ACPA positive (n (\%)) & $0(0)$ & $8(62)$ & $7(50)$ \\
ACPA level* (kAU/l) (median (IQR)) & - & $224(136-685)$ & $678(119-2443)$ \\
IgM-RF and ACPA both pos (n (\%)) & $0(0)$ & $0(0)$ & $5(36)$ \\
ESR (mm/h) (median (IQR)) & - & $7(2-14) \dagger$ & $12(8-14)$ \\
CRP (mg/l) (median (IQR)) & $0.9(0.5-4.4)$ & $1.6(0.6-6.1)$ & $5.6(1.1-14.4)$ \\
$68 \mathrm{TJC}(\mathrm{n})$ (median (IQR)) & $0(0)$ & $2(1-5)$ & $13(4-27)$ \\
$66 \mathrm{SJC}(\mathrm{n})$ (median (IQR)) & $0(0)$ & $0(0)$ & $7(4-11)$ \\
\hline
\end{tabular}

Categorical variables: $\mathrm{n}(\%)$. Continuous variables (data not normally distributed): median (IQR).

*Levels only in positive patients.

tLevel missing from one individual.

ACPA, anticitrullinated protein antibodies; ESR, erythrocyte sedimentation rate; CRP, C-reactive protein; IgM-RF, IgM rheumatoid factor; 68 TJC, tender joint count of 68 joints; $66 \mathrm{SJC}$, swollen joint count of 66 joints.

cell suspension. Subsequently, cells were washed with phosphate buffered saline (PBS) containing $0.01 \% \mathrm{NaN}_{3}$ and $0.5 \%$ BSA. Cells were stained for $30 \mathrm{~min}$ at $4^{\circ} \mathrm{C}$ and protected from light using the following directly labelled antibodies: CD3 FITC (Sanquin, Amsterdam, The Netherlands), CD45 V500, CD69 PerCP, CD27 PerCP-Cy5.5, IgD FITC (BD Biosciences, Breda, The Netherlands), CD19 eFluor 450, CD4 Pe-Cy7, CD45RO PE, CD45RA eFluor 450 and CD8 APC eFluor 780
(eBioscience). After incubation, cells were washed and measured on a FACS CANTO II (BD Biosciences). Data were analysed using FlowJo software (Tree Star, Ashland, Oregon, USA).

\section{Statistics}

Not normally distributed data were presented as medians (IOR). Differences between study groups were analysed using one-way analysis of variances with post-Bonferroni's multiple
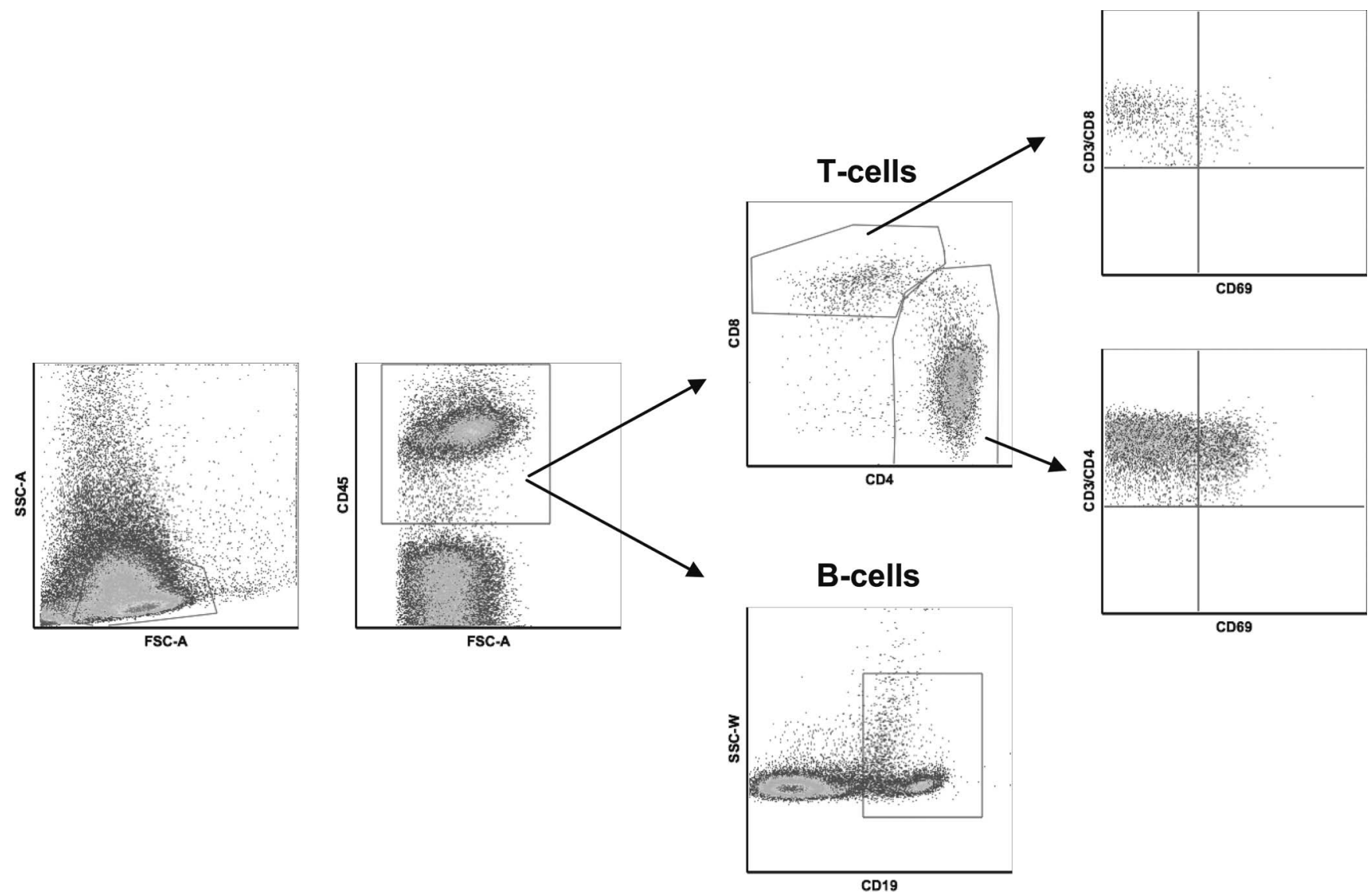

Figure 1 Flow cytometry gating strategy. Fresh lymph node biopsies were immediately processed for flow cytometry analysis using T-cell surface markers including CD3, CD4, CD8, CD45 and CD69, and B-cell surface marker CD19. This figure shows the regions used to identify the different cell populations. 
comparison tests or unpaired $t$ test where appropriate. Categorical data were presented as numbers (percentages), and differences between groups were analysed using $\chi^{2}$ test. GraphPad Prism Software (V.5, GraphPad Software, La Jolla, California, USA) was used for statistical analysis.

\section{RESULTS}

Lymph node biopsies were obtained from 13 IgM-RF and/or ACPA positive individuals without arthritis. Nine of them were referred to the outpatient rheumatology clinic because of joint pain, and four through testing first-degree relatives of RA patients (two of the latter did not have arthralgia, all

A

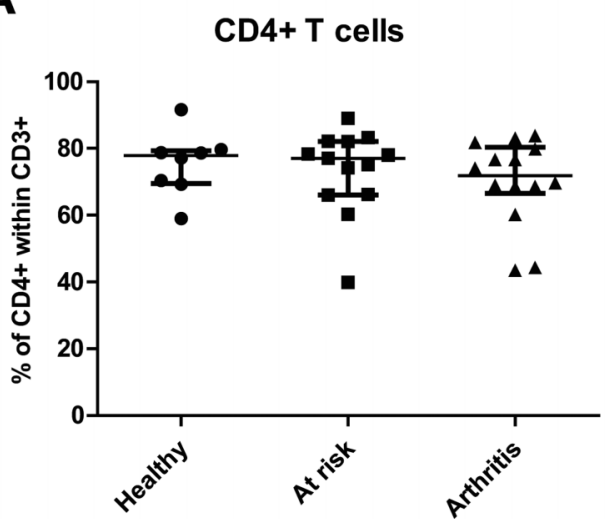

C CD4+CD69+ activated T cells

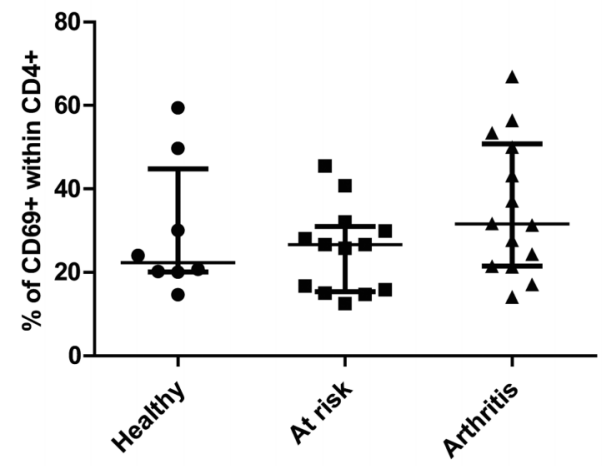

E

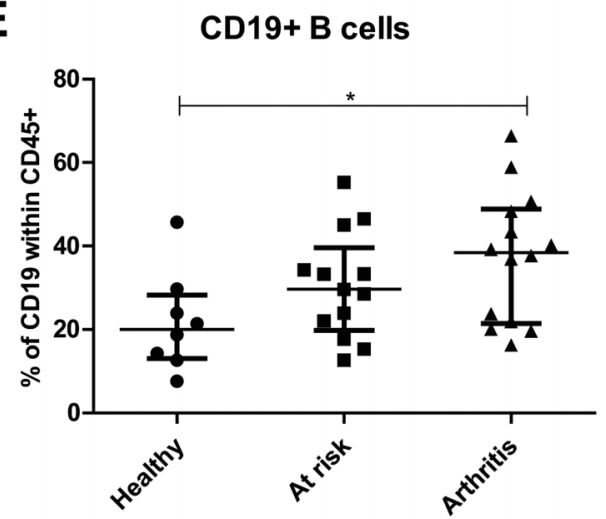

other individuals did have arthralgia). None of these autoantibody-positive individuals has developed arthritis yet after a follow-up time of median (IOR) 12 (9-14) months. Additional characteristics are given in online supplementary table S1. For comparison, lymph node biopsies were obtained from 14 early arthritis patients (eight RA according to the 2010 ACR/EULAR criteria for RA and six unclassified arthritis (UA) of whom four fulfilled 2010 ACR/EULAR criteria for RA after follow-up; median (IOR) arthritis duration one (0-2) months) and eight autoantibody-negative healthy controls. Table 1 shows the demographic data of the study participants.

B

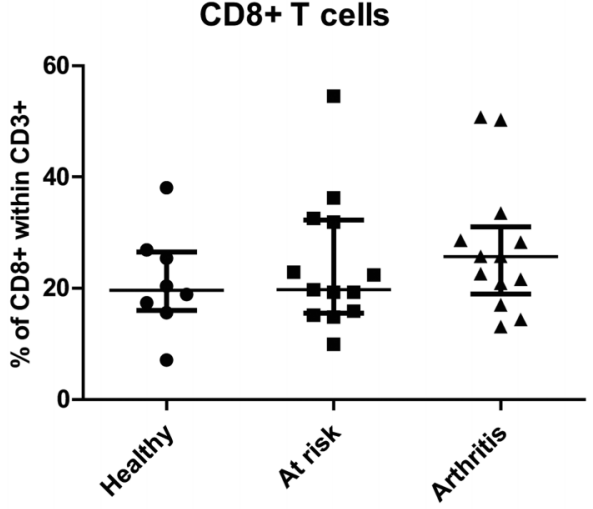

D CD8+CD69+ activated T cells

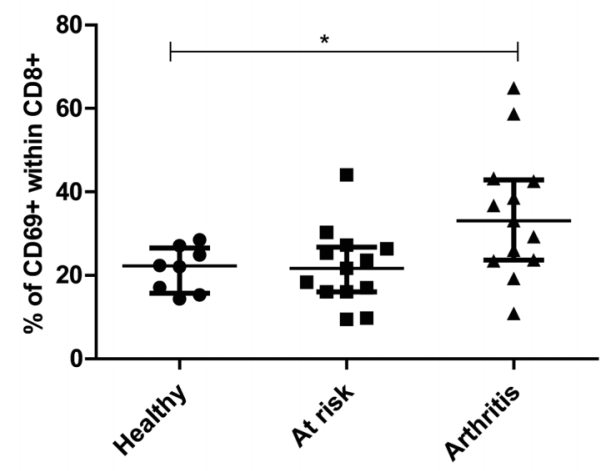

F CD19+ B cells in early arthritis

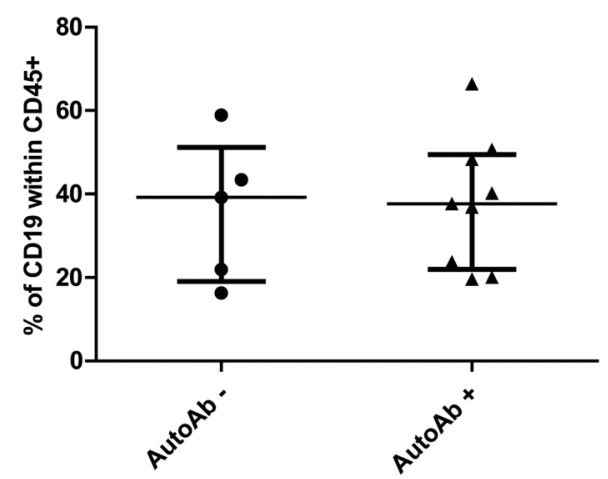

Figure 2 T-cell and B-cell frequencies in lymph node biopsies. Fresh lymph node biopsies were immediately processed for flow cytometry analysis as explained in figure 1. For interindividual comparisons frequencies were determined as \% of CD45 cells. The frequency of CD4 (A) and CD8 (B) T cells were as expected and not different between the three study groups. The frequency of activated CD4CD69 T cells (C) was highly variable between subjects, but significantly more activated CD8CD69 T cells (D) were observed in lymph node samples from patients with arthritis compared with healthy controls. The frequency of CD19 B cells was significantly increased in patients with arthritis compared with healthy controls (E). The number of CD19 B cells was not dependent of autoantibody (autoAb) status (F). 
To explore the cellular composition of the lymph node compartment, freshly collected lymph node specimens were directly analysed by multicolour flow cytometry for the presence of specific $\mathrm{T}$ and $\mathrm{B}$ lymphocytes including their activation or differentiation state (figure 1). The frequencies of CD4 and CD8 $\mathrm{T}$ cells were within the expected range $(\sim 80 \%$ CD4 and $\sim 20 \%$ CD8 within CD3 T cells) and no differences were observed between at risk individuals, early arthritis patients and healthy controls (figure 2A,B). Subsequently, the percentage of activated $\mathrm{T}$ cells was determined by analysing coexpression of CD69 (figure 2C,D). Interestingly, the percentage of activated CD8 $\mathrm{T}$ cells was different between the three study groups $(p=0.012)$, and specifically increased in early arthritis patients compared with healthy controls (median (IOR) 33.10 (23.60$42.905)$ vs $22.20(15.75-26.55), \mathrm{p}<0.05))$. The increase in percentage of activated $\mathrm{T}$ cells was not dependent on the presence of autoantibodies or the presence of arthritis in the lower limb on the ipsilateral side of the biopsied lymph node (data not shown). In addition, there were no differences between UA and RA patients.

Next, we analysed the percentage of CD19 B cells which showed a significant difference between the three study groups $(p=0.049)$. The percentage of CD19 B cells was significantly higher in early arthritis patients compared with healthy controls (median (IOR) 38.45 (21.45-48.90) vs 20.05 (13.03-28.25), $\mathrm{p}<0.05$ ) (figure $2 \mathrm{E}$ ). We also observed a trend towards increased CD19 B cells in at risk individuals compared with healthy controls (median (IOR) 29.60 (19.85-39.65) vs 20.05 (13.03-28.25), $p>0.05)$. The increased number of $B$ cells was independent of the presence of autoantibodies (figure $2 \mathrm{~F}$ ). In the group of early arthritis patients, no differences were observed between UA and RA patients, and the increased number of $B$ cells was independent of the presence of arthritis in the lower limb on the ipsilateral side of the biopsied lymph node (data not shown). The percentage of different subsets of $B$ cells, naïve, memory switched and memory non-switched (based on IgD and CD27), was comparable between the different study groups (data not sown).

\section{DISCUSSION}

This explorative study was undertaken to explore for the first time the cellular composition of lymph nodes in at risk individuals having systemic autoimmunity associated with RA, and early arthritis patients compared with healthy controls. First, the results indicate that flow cytometry analysis of lymph node biopsies is a feasible method for studying the cellular composition and activation of lymph node tissue in the earliest phases of arthritis. Second, we observed more CD19 B cells and activated CD8 T cells in early arthritis patients compared with healthy controls. Third, there was a trend towards an increase in CD19 B cells in at risk individuals compared with healthy controls.

During an immune response, the egress of $T$ lymphocytes from lymph nodes is shut down transiently by downregulation of S1P1 and upregulation of CD69 leading to lymphocyte retention, maturation and proliferation. ${ }^{16}$ The results of this explorative study suggest increased activation of $\mathrm{T}$ cells, as shown by coexpression of CD69, within lymph nodes of arthritis patients during the earliest phase of disease. Of interest, the percentage of activated CD8 $\mathrm{T}$ cells is increased. These results are in line with animal models of arthritis where a skewed $\mathrm{CD} 4 / \mathrm{CD} 8$ ratio is observed in regional lymph nodes before the onset of arthritis. ${ }^{13} 14$ These results support the notion that $\mathrm{T}$ cells are intimately involved in the initiation of seropositive RA. ${ }^{17-19}$ Future research should focus on the identification of $\mathrm{T}$-cell subset(s) and antigen specificity associated with development of RA.

Of interest, an increased percentage of CD19 B cells was observed in lymph nodes of early arthritis patients and autoantibody-positive subjects at risk of developing RA. These results of the lymph node analyses differ from those in a previous study on peripheral blood, in which gene expression profiling of at risk individuals revealed a low B-cell signature especially in those individuals who developed arthritis after follow-up. ${ }^{20}$ It is tempting to speculate that the $\mathrm{B}$ cells are retained in the lymph nodes, to ensure maturation and differentiation during the immune response which would be in line with findings in animal models of arthritis. ${ }^{12} 13$

An obvious limitation of the current study is the small sample size and the short follow-up period of the 'at risk' individuals. Of note, it has been challenging to obtain lymph node biopsy samples from these patients and controls. We now have all the tools available to first identify those individuals at risk of developing arthritis and to prospectively analyse the immune cells in lymph node tissues. This will create a framework for studying the molecular events taking place in lymph nodes before the onset of arthritis that can be potentially related to the processes involved in the pathogenic changes in synovial tissues.

Acknowledgements We thank our study subjects for participating in the study, the department of radiology for the lymph node sampling, the AMC KIR lab technicians for sample processing and Johan Dobber for his advice on flow cytometry on lymph node needle biopsies.

Contributors LGMvB and MJHdH were involved in study design, data collection and analysis, interpretation of the results and writing of the manuscript; both contributed equally to the manuscript. THR was involved in data collection and analysis, interpretation of the results and writing of the manuscript. IJAJZ was involved in data collection, interpretation of the results and revising the manuscript. MM was involved in study design, data collection, interpretation of the results and revising the manuscript. DMG was involved in study design, data collection, interpretation of the results and revising the manuscript. PPT was involved in study design, interpretation of the results and revising the manuscript.

Funding This study was sponsored by the Dutch Arthritis Foundation grants 08-1-310 and 11-1-308, The Netherlands Organisation for Health Research and Development (ZonMw) Veni project 916.12.109, and the IMI EU-funded project BeTheCure no. 115142.

Competing interests None.

Ethics approval The medical ethical committee of the Academic Medical Center Amsterdam approved this study.

Provenance and peer review Not commissioned; externally peer reviewed.

Open Access This is an Open Access article distributed in accordance with the Creative Commons Attribution Non Commercial (CC BY-NC 3.0) license, which permits others to distribute, remix, adapt, build upon this work non-commercially, and license their derivative works on different terms, provided the original work is properly cited and the use is non-commercial. See: http://creativecommons.org/ licenses/by-nc/3.0/

\section{REFERENCES}

1. Ouinn MA, Conaghan PG, Emery P. The therapeutic approach of early intervention for rheumatoid arthritis: what is the evidence? Rheumatology (Oxford) 2001;40:1211-20.

2. Mottonen T, Hannonen $\mathrm{P}$, Korpela $\mathrm{M}$, et al. Delay to institution of therapy and induction of remission using single-drug or combination-disease-modifying antirheumatic drug therapy in early rheumatoid arthritis. Arthritis Rheum 2002;46:894-8.

3. O'Dell JR. Treating rheumatoid arthritis early: a window of opportunity? Arthritis Rheum 2002;46:283-5.

4. Nielen MM, van Schaardenburg D, Reesink HW, et al. Specific autoantibodies precede the symptoms of rheumatoid arthritis: a study of serial measurements in blood donors. Arthritis Rheum 2004;50:380-6. 
5. Rantapaa-Dahlqvist S, de Jong BA, Berglin E, et al. Antibodies against cyclic citrullinated peptide and IgA rheumatoid factor predict the development of rheumatoid arthritis. Arthritis Rheum 2003;48:2741-9.

6. Aho K, Palosuo T, Raunio V, et al. When does rheumatoid disease start? Arthritis Rheum 1985;28:485-9.

7. Aho K, von ER, Kurki P, et al. Antikeratin antibody and antiperinuclear factor as markers for subclinical rheumatoid disease process. J Rheumatol 1993;20:1278-81

8. Jorgensen KT, Wiik A, Pedersen $M$, et al. Cytokines, autoantibodies and viral antibodies in premorbid and postdiagnostic sera from patients with rheumatoid arthritis: case-control study nested in a cohort of Norwegian blood donors. Ann Rheum Dis 2008;67:860-6.

9. Bos WH, Wolbink GJ, Boers M, et al. Arthritis development in patients with arthralgia is strongly associated with anti-citrullinated protein antibody status: a prospective cohort study. Ann Rheum Dis 2010;69:490-4.

10. Gerlag DM, Raza K, van Baarsen LG, et al. EULAR recommendations for terminology and research in individuals at risk of rheumatoid arthritis: report from the Study Group for Risk Factors for Rheumatoid Arthritis. Ann Rheum Dis 2012;11:638-41.

11. van de Sande MG, de Hair MJ, van der Leij $C$, et al. Different stages of rheumatoid arthritis: features of the synovium in the preclinical phase. Ann Rheum Dis 2011;70:772-7.

12. Li J, Kuzin I, Moshkani S, et al. Expanded CD23(+)/CD21 (hi) B cells in inflamed lymph nodes are associated with the onset of inflammatory-erosive arthritis in
TNF-transgenic mice and are targets of anti-CD20 therapy. J Immunol 2010;184:6142-50

13. Rodriguez-Palmero M, Pelegri C, Ferri MJ, et al. Alterations of lymphocyte populations in lymph nodes but not in spleen during the latency period of adjuvant arthritis. Inflammation 1999:23:153-65.

14. Wooley PH, Whalen JD. Pristane-induced arthritis in mice. III. Lymphocyte phenotypic and functional abnormalities precede the development of pristane-induced arthritis. Cell Immunol 1991;138:251-9.

15. de Hair MJ, Zijlstra IA, boumans MJ, et al. Hunting for the pathogenesis of rheumatoid arthritis: core-needle biopsy of inguinal lymph nodes as a new research tool. Ann Rheum Dis 2012;71:1911-2.

16. Schwab SR, Cyster JG. Finding a way out: lymphocyte egress from lymphoid organs. Nat Immunol 2007:8:1295-301.

17. Firestein GS, Zvaifler NJ. How important are T cells in chronic rheumatoid synovitis?: II. T cell-independent mechanisms from beginning to end. Arthritis Rheum 2002;46:298-308.

18. Fox DA. The role of T cells in the immunopathogenesis of rheumatoid arthritis: new perspectives. Arthritis Rheum 1997:40:598-609.

19. Cope AP. T cells in rheumatoid arthritis. Arthritis Res Ther 2008;10(Suppl 1):S1.

20. van Baarsen LG, Bos WH, Rustenburg F, et al. Gene expression profiling in autoantibody-positive patients with arthralgia predicts development of arthritis. Arthritis Rheum 2010;62:694-704. 